\title{
What Sequences on High-Field MR Best Depict Temporal Resolution of Experimental ICH and Edema Formation in Mice?
}

\author{
Mingchang Li, ${ }^{1,2}$ Reza M. Akhavan-Sharif, ${ }^{3}$ Robert M. Friedlander, ${ }^{1,4}$ \\ Rose Du, ${ }^{1}$ and Ruth Thiex ${ }^{1,5}$ \\ ${ }^{1}$ Department of Neurosurgery, Brigham and Women's Hospital, Harvard Medical School, Boston, MA 02115, USA \\ ${ }^{2}$ Department of Neurosurgery, The Second Affiliated Hospital of Guangzhou Medical University, Guangzhou 510260, China \\ ${ }^{3}$ Department of Radiology, Beth Israel Deaconess Medical Center, Boston, MA 02115, USA \\ ${ }^{4}$ Department of Neurological Surgery, University of Pittsburgh Medical Center, Pittsburgh, PA 15213, USA \\ ${ }^{5}$ Division of Endovascular Neurosurgery and Interventional Neuroradiology, Departments of Radiology and Neurosurgery, \\ Brigham and Women's Hospital, Harvard Medical School, Boston, MA 02115, USA
}

Correspondence should be addressed to Ruth Thiex, ruth.thiex@post.rwth-aachen.de

Received 3 January 2012; Accepted 27 February 2012

Academic Editor: Oreste Gualillo

Copyright (c) 2012 Mingchang Li et al. This is an open access article distributed under the Creative Commons Attribution License, which permits unrestricted use, distribution, and reproduction in any medium, provided the original work is properly cited.

\begin{abstract}
Background and Purpose. Pilot study to examine the use of T1-, T2-, and T2*-weighted images for evaluating hematoma size and extent of edema in mouse brain at high field. Methods. Following collagenase-induced intracerebral hemorrhage, nine mice were imaged at 4.7 T using T1-, T2-, and T2*-weighted images for hematoma and edema quantitation on days 1, 3, 10, and 21 after surgery. Values were compared with morphometric analysis of cryosections at the time of final MR imaging. Results. For hematoma quantitation, the Spearman correlation coefficient $(r)$ between T1 signal change and histology was $0.70(P<0.04)$ compared with $r=0.61(P<0.09)$ for T2*. The extent of perihematomal edema formation on cryosections was well reflected on T2 with $r=0.73$ $(P<0.03)$. Conclusions. Within the limits of our pilot study, MR imaging on $4.7 \mathrm{~T}$ appears to approximate the temporal changes in hematoma and edema sizes in murine ICH well, thus laying the groundwork for longitudinal studies on hematoma resorption and edema formation.
\end{abstract}

\section{Introduction}

In humans, intracerebral hemorrhage (ICH) causes secondary damage in the brain through the induction of cerebral edema and perihematomal injury $[1,2]$. Perihematomal edema with mass effect is an almost universal complication of ICH, elaborated over several days after the initial insult. Glutamate release, bioenergetic failure, inflammation, and apoptosis play a key role in the pathogenesis of secondary injury $[3,4]$. In animal models of $\mathrm{ICH}$, molecular and cellular studies also indicate that hemorrhage induces inflammation, apoptotic cell death, and progressive tissue destruction in perihematomal tissue [5-7].

Various knockout mice have been bred to study the gene expression of the pro-oxidants heme [8] and ferrous iron [9], proteolytic enzymes such as metalloproteinases [10], thrombin [10], and inducible nitric oxide synthase [11], as well as leucocyte adhesion molecules (CD18) [12] in the perihematomal area. Their genetic variability has turned the mouse into a key study animal for intracerebral hemorrhage despite the small size of its brain. The hematoma is either induced by stereotactic injection of collagenase or autologous blood into the basal ganglia. Longitudinal studies of hematoma kinetics in mice are sparse and generally use one modality only [13]. The purpose of our pilot study was (i) to develop T1-and T2-weighted MRI protocols for highresolution studies of mouse brain in vivo which allow for the identification of hematoma and edema, and (ii) to perform a direct comparison of hematoma and edema volumes on MRI with morphometric studies on cryosections. Serial MR imaging would allow longitudinal studies of the temporal and spatial evolution of intracerebral hemorrhages and perihematomal injury. That would not only substantially reduce the amount of animals needed, but also better depict 
the time-dependent action mechanism of various pathogenetic factors.

\section{Materials and Methods}

2.1. Animals. C57BL/6 mice (Jackson Laboratories, Bar Harbor, ME, USA) were maintained in the Department of Laboratory Animal Research at Harvard Medical School with access to food and water ad libitum. All experiments were performed in accordance to the National Institutes of Health guide for the care and use of laboratory animals as well as the institutional guidelines established by the Institutional Animal Care and Use Committee (IACUC) at Harvard Medical School.

2.2. Intracerebral Hemorrhage Model. The procedure of hematoma induction was previously described [14-17]. A total of 11 male adult mice, 10-12 weeks of age, with a body weight of $20-30 \mathrm{~g}$, were used. Mice were anesthetized by intraperitoneal injection of $0.5 \mathrm{mg} / \mathrm{g}$ body weight of avertin (tribromoethanol, Sigma-Aldrich, St. Louis, MO, USA). To induce hemorrhage, mice were injected with $0.075 \mathrm{U}$ collagenase type VII-s (Sigma-Aldrich, St. Louis, MO, USA) in $500 \mathrm{~nL}$ saline into the left caudate putamen using the following stereotactic coordinates: $0.5 \mathrm{~mm}$ posterior, $3.0 \mathrm{~mm}$ lateral to the bregma, and $4.0 \mathrm{~mm}$ in depth. Collagenase was infused over 2 minutes using a stereotaxic injector (Stoelting, Wood Dale, IL, USA) and the $1 \mu \mathrm{L}$-syringe (Hamilton, Reno, $\mathrm{NV}$ ) stayed in place for 10 minutes to prevent reflux.

2.3. MRI Setup. This study was performed in 2 parts. In part 1, two mice underwent MR imaging 2, 8, and 24 hours after collagenase injection to define the best early time points for MR imaging. In part 2, nine mice were subjected to an imaging schedule from days $1(n=9), 3(n=7), 10(n=5)$, and $21(n=2)$ based on the different phases of edema (hyperacute, acute, subacute, chronic) that bear their own pathophysiology. Animals were evaluated using a modified 28-point neurological scoring system before isoflurane anesthesia for the MR [14]. The test included body symmetry, gait, climbing, circling behaviours, front limb asymmetry, and compulsory circling. Each point was graded from 0 to 4 , establishing a maximum deficit score of 24 . MR imaging was performed with a high-field 4.7 Tesla MR scanner (Biospin, Bruker, Germany) at the Center for Basic MR Research at Beth Israel Deaconess Medical Center. Anesthesia was induced with $2 \%$ isoflurane and maintained with $1.6 \%$ isoflurane in $100 \% \mathrm{O}_{2}$ via an MR-compatible nose cone apparatus to minimize motion artifacts. Mice were positioned headfirst and prone inside a plexiglass "cradle" with respiratory monitoring and a constant flow of isoflurane delivered directly to the nose of the mouse. This method bypasses intubation of the vulnerable trachea. The magnet compatible apparatus allows a precise and reproducible fixation of the head at the center of the Bruker linear birdcage radiofrequency coil with an inner diameter of $22 \mathrm{~mm}$. After the isoflurane administration was discontinued, mice were monitored until awake from anesthesia.
2.4. MRI Parameters. T1- and T2-weighted images were acquired at a $256 \times 256$ matrix and a $2.56 \times 2.56 \mathrm{~cm}^{2}$ field of view. A relaxation enhancement (RARE) sequence was used for both T1-weighted (echo time/relaxation time $[\mathrm{TE} / \mathrm{TR}]=$ 12/500, RARE factor $=1)$ and T2-weighted $(\mathrm{TE} / \mathrm{TR}=75 /$ 2000, RARE factor $=12$ ) imaging. T2*-weighted imaging parameters were $\mathrm{TE}=14 \mathrm{~ms}, \mathrm{TR}=1500 \mathrm{~ms}, \mathrm{FOV}=2.56 \times$ $2.56 \mathrm{~cm}^{2}$, and $\mathrm{FA}=30^{\circ}$, matrix $128 \times 128$. In all acquisitions, 9 axial slices with $1 \mathrm{~mm}$ thickness were obtained. Hematoma size was determined from $\mathrm{T} 1$ and $\mathrm{T} 2 *$ images, and $\mathrm{T} 2$ images were used for edema quantification. The measurements were performed by an examiner blinded to the exam date using a computer-assisted image analysis program (ImageJ, NIH, available at website http://rsb.info.nih.gov/ij/). The values for hematoma and edema volumes were compared with those determined from cryosections.

2.5. Histology. Following final MR imaging, brains were removed, placed in $4 \%$ paraformaldehyde and cryoprotected in $30 \%$ sucrose for 3 days at $4^{\circ} \mathrm{C}$ before they were frozen. Coronal sections of $20 \mu \mathrm{m}$-thickness were taken at $300 \mu \mathrm{m}$ intervals over the rostral-caudal extent of the lesion. Sections were stained with Luxol Fast Blue and cresyl violet according to previously published protocols $[15,17,18]$. Hematoma and edema volumes were measured by digitally analyzing stained sections with ImageJ. Lesion volumes $\left(\mathrm{mm}^{3}\right)$ were computed as running sums of lesion area multiplied by the thickness of each section $(300 \mu \mathrm{m})$ over the extent of the lesion expressed as an orthogonal projection [19].

2.6. Statistical Analysis. Given the small sample size at different examination dates, the correlation coefficient (Spearman coefficient) for non-parametric correlations and two-tailed $\mathrm{p}$ values at a confidence interval $\mathrm{CI}=95 \%$ were analyzed using one-way ANOVA.

\section{Results}

3.1. MR Appearance of Hematoma and Edema. In two animals, MR imaging was performed as early as 2 hours after hematoma induction, and the hematoma was observed as a focal hypointensity on T1-, T2-, and T2*-weighted images. The hematoma remained stable without any evidence of expansion when scanned again 8 hours and 24 hours following collagenase injection (Figure 1).

In mice with serial MR imaging up to 72 hours, 10 and 21 days, the predominantly low-signal hematoma within the left caudate/putamen in T1-, T2-, and T2*-weighted images were consistent with deoxyhemoglobin and intracellular methemoglobin after 24 hours. The signal converted to predominantly high intensity at 72 hours to 10 days on T2weighted RARE spin-echo images, consistent with extracellular methemoglobin (Figure 2). The initially hypointense hematoma on T1-weighted images became hyperintense on days 3 and 10. Even on T2*-weighted images, the signal of the hematoma core became hyperintense at 10 days. On day 21 , a low-signal streak was left at the site of the former spaceoccupying hematoma on T1-, T2-, and T2*-weighted images (Figures 3 and 4). 


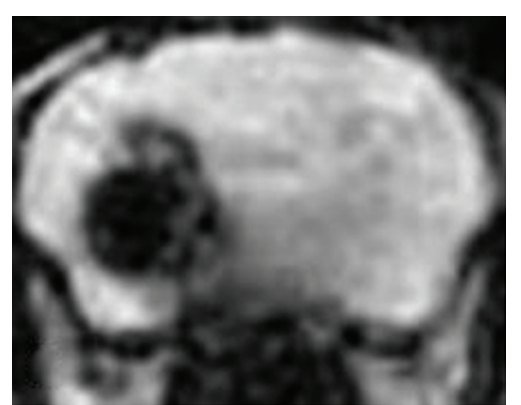

(a)

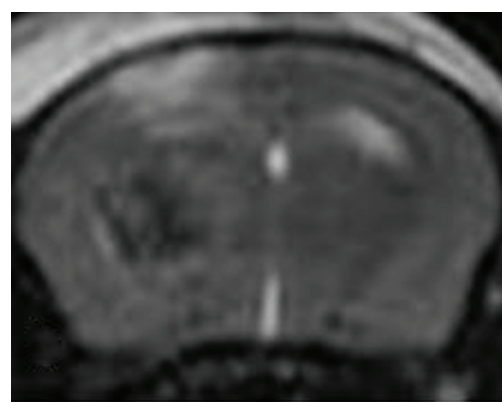

(b)

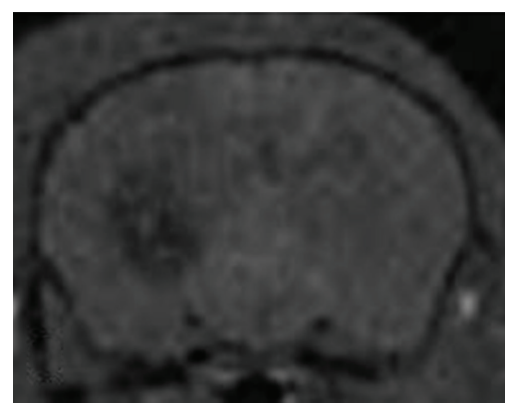

(c)

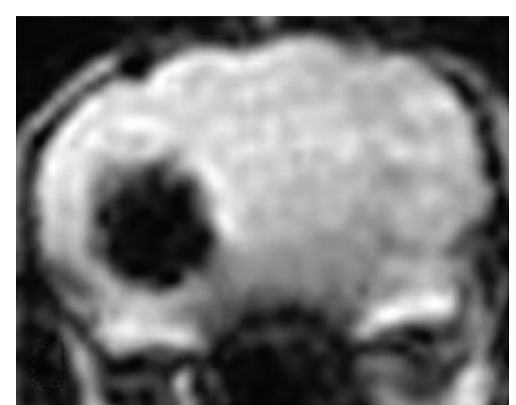

(d)

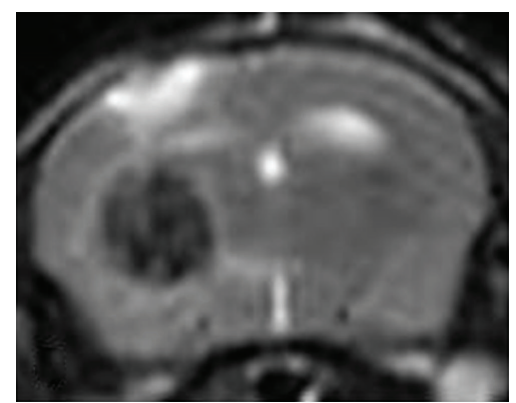

(e)

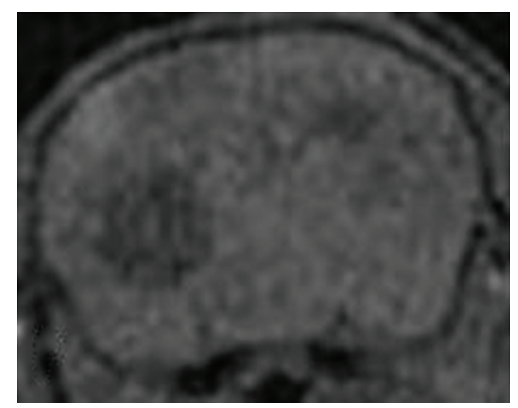

(f)

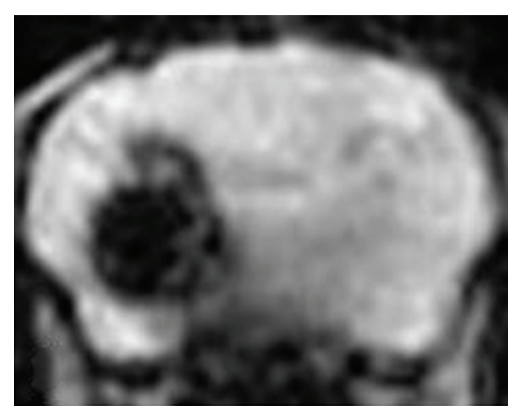

(g)

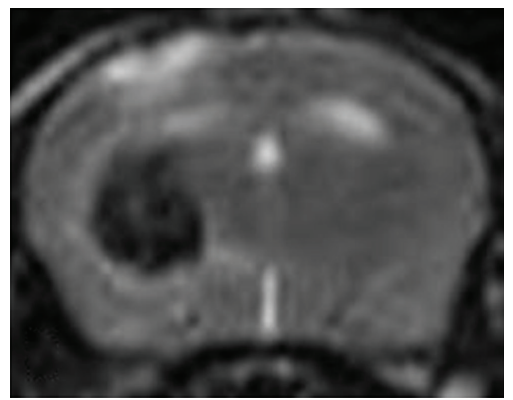

(h)

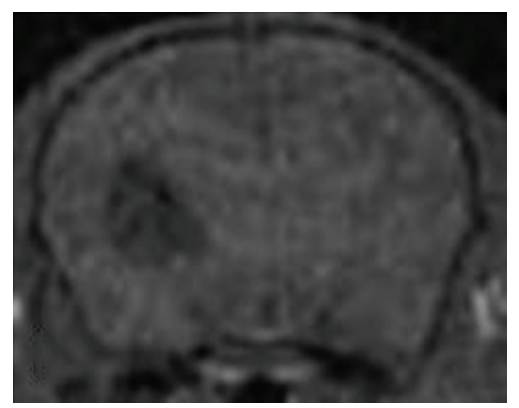

(i)

Figure 1: MR tomographic images on $4.7 \mathrm{~T}$ using T2*- (a, d, and g), T2- (b, e, and h), and T1-weighted images (c, f, and i) in a mouse 2 $(\mathrm{a}-\mathrm{c}), 8(\mathrm{~d}-\mathrm{f})$, and 24 hours $(\mathrm{g}-\mathrm{i})$ after stereotactic collagenase injection. The hematoma appears hypointense on T1 as early as 2 hours after collagenase injection and does not change in signal intensity and homogeneity over the first 24 hours. The blooming effect of T2*-weighted sequences makes the hematoma appear larger and needs referencing with T1- and T2-weighted images. T2-weighted images reveal a rim of hyperintensity surrounding the hypointense core as early as 8 hours. There is no evidence of hematoma enlargement over the first 24 hours.

\subsection{Quantification of Hematoma and Edema Volumes.}

Table 1 illustrates the mean hematoma sizes on T1- and $\mathrm{T} 2 *$-weighted images and the mean edema sizes on T2 on day 1 for nine mice, on day 3 for seven mice, on day 10 for four mice, and for the remainder $(n=2)$ on day 21 . After final imaging, the equivalent hematoma and edema volumes were compared with the hematoma and edema areas on histology as also shown in Table 1. The Spearman correlation coefficient between hematoma volume on T2* weighted images and histology was $r=0.61(P<0.09)$ over all times points, thus not reaching statistical significance. The Spearman correlation coefficient between hematoma volume on T1 and histology, however, was statistically significant $r=0.7(P<0.04)$. So was the coefficient for edema volume on T2 and histology on all examination dates with $r=0.73$ $(P<0.03)$.

\section{Discussion}

Ongoing research in intracerebral hemorrhage and the prospects of therapy have raised the need for a noninvasive method to investigate ICH in rodent models. The quantification of hematoma volume is currently based on spectrophotometry assays $[8,20]$, histomorphometry on stained brain slices [15], or computer-assisted outlining of brain slices [20], thus excluding follow-up measurements during the disease progression and for the evaluation of novel therapeutic interventions. The same accounts for edema volumetry that uses brain water content measurements [11, 16, 17], blood-brain barrier permeability for Evans blue dye on histology [11], or hemispheric enlargement [8] for quantitation. Among the biological imaging techniques, magnetic resonance imaging (MRI) constitutes an excellent tool for 


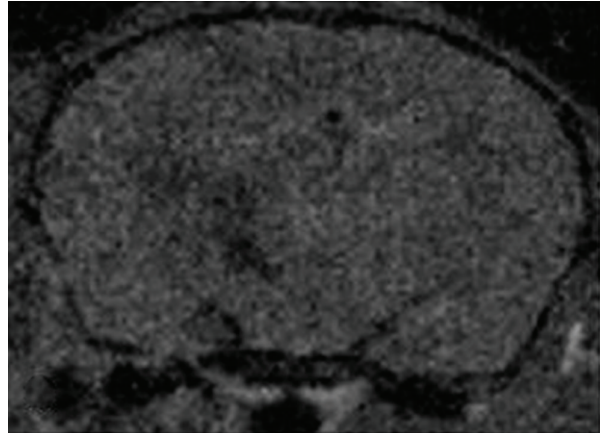

(a)

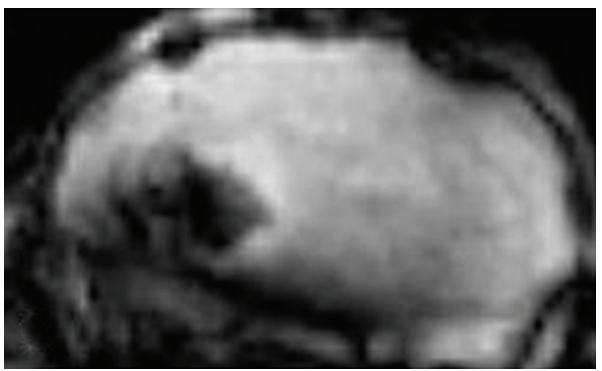

(c)

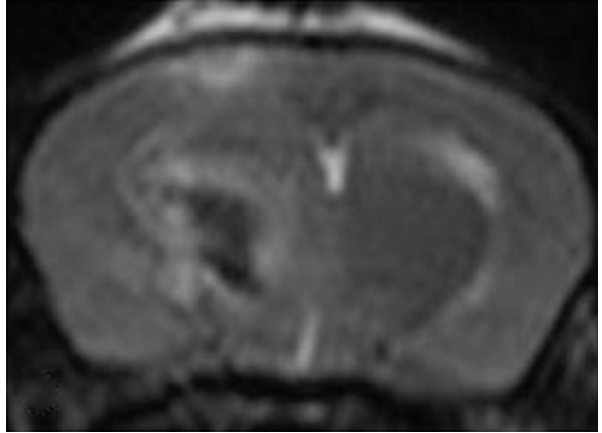

(b)

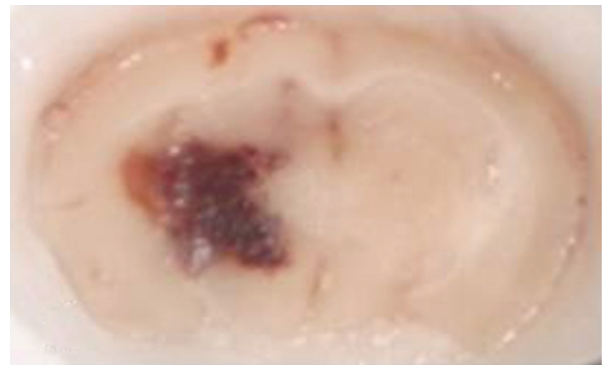

(d)

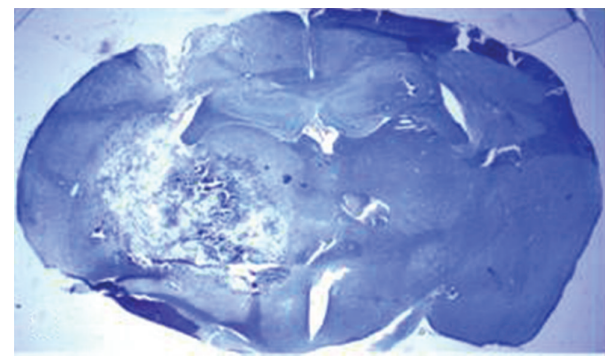

(e)

FIGURE 2: T1- (a), T2- (b), and T2* - (c) weighted images in a mouse 24 hours after hematoma induction reveal a hypointensity that matched well with the hematoma size found on frozen section (d) and cryostat section stained with Luxol Fast Blue and cresyl violet [ $\times 10](e)$. Again, T2*-weighted images need referencing with T1- and T2-weighted images for hematoma quantitation. Of note is an artefact on the left to midventral surface $(c)$.

TABLE 1: MR volumetry and histology.

\begin{tabular}{|c|c|c|c|c|c|c|}
\hline Study dates & $\begin{array}{c}\text { Mean hematoma } \\
\text { size } \pm \mathrm{SD} \text { on } \mathrm{T} 1 \text { in } \\
\mathrm{mm}^{3}\end{array}$ & $\begin{array}{l}\text { Mean hematoma } \\
\text { size } \pm \mathrm{SD} \text { on } \mathrm{T}^{*} \\
\text { in } \mathrm{mm}^{3}\end{array}$ & $\begin{array}{l}\text { Mean edema } \\
\text { size } \pm \text { SD on T2 } \\
\text { in } \mathrm{mm}^{3}\end{array}$ & $\begin{array}{c}\text { Mean edema size } \pm \mathrm{SD} \\
\text { on } \mathrm{T} 2 \text { in } \mathrm{mm}^{3} \text { for } \\
\text { euthanized mice }\end{array}$ & $\begin{array}{l}\text { Mean edema size } \pm \mathrm{SD} \\
\text { on histology in } \mathrm{mm}^{3} \\
\text { for euthanized mice }\end{array}$ & $\begin{array}{c}\text { Median } \\
\text { neurological } \\
\text { score }(0-24) \\
\end{array}$ \\
\hline Day 1 & $14.0 \pm 3.4$ & $13.4 \pm 3.4$ & $11.1 \pm 5.6$ & $16.0 \pm 4.0$ & $10.6 \pm 1.5$ & 4 \\
\hline Day 3 & $7.4 \pm 3.8$ & $8.0 \pm 4.4$ & $8.9 \pm 3.4$ & $12.2 \pm 1.3$ & $27.2 \pm 2.2$ & 2 \\
\hline Day 10 & $6.1 \pm 3.4$ & $6.9 \pm 3.5$ & $1.6 \pm 0.8$ & $1.4 \pm 0.6$ & $1.5 \pm 1.6$ & 0 \\
\hline Day 21 & $3.4 \pm 3.1$ & $4.3 \pm 0.9$ & $0.8 \pm 1.1$ & $0.8 \pm 1.1$ & $0.1 \pm 0.1$ & 0 \\
\hline
\end{tabular}

neuroimaging in rats [21] and pigs [22, 23]. Owing to the variety of knockout genotypes in mice, repeated studies of the same animal are important research tools to study hematoma and edema evolution in a longitudinal fashion. Previous MRI studies of the mouse brain have either been postmortem imaging of excised specimen with a nearmicroscopic spatial resolution $[24,25]$ or in vivo studies with a high in-plane resolution at the expense of a much poorer section thickness [26].

At high magnetic fields, the ratio between surface and volume in the head is important because of the presence of high susceptibility effects and poor magnetic field homogeneity in the whole head. Even with optimization of the shimming procedure and a reduction in TR, it was not 


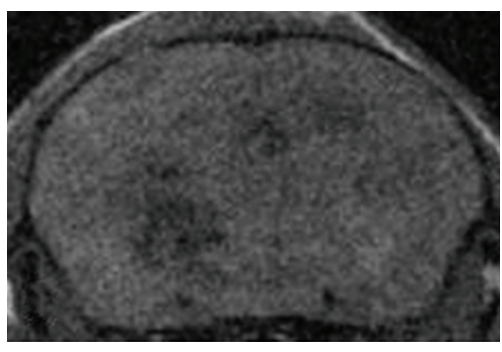

(a)

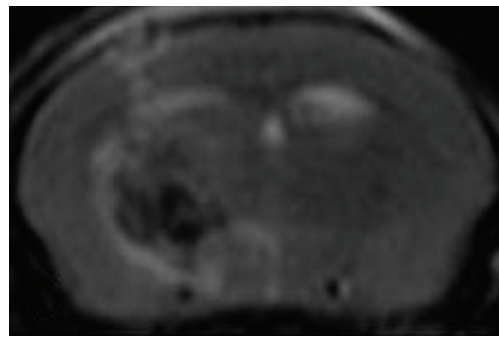

(b)

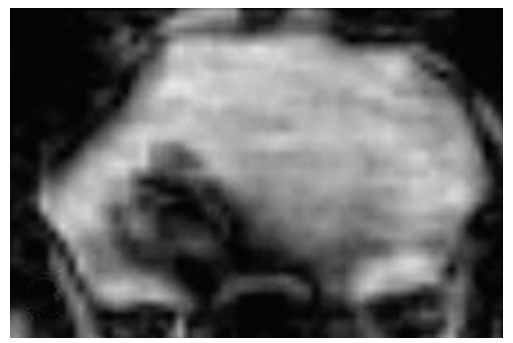

(c)

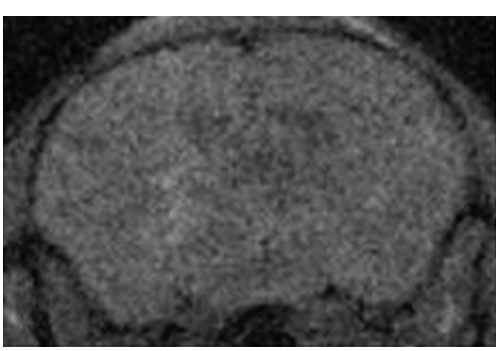

(d)

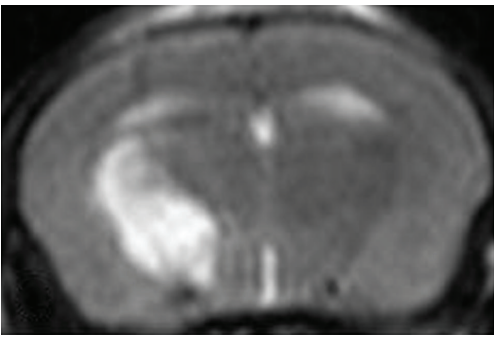

(e)

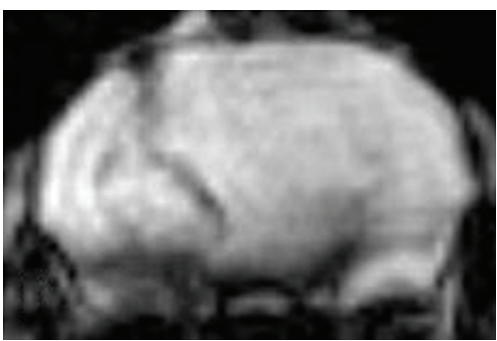

(f)

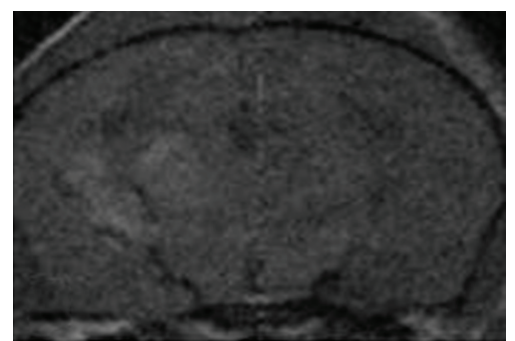

(g)

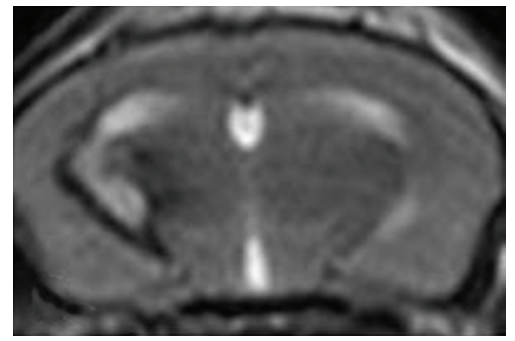

(h)

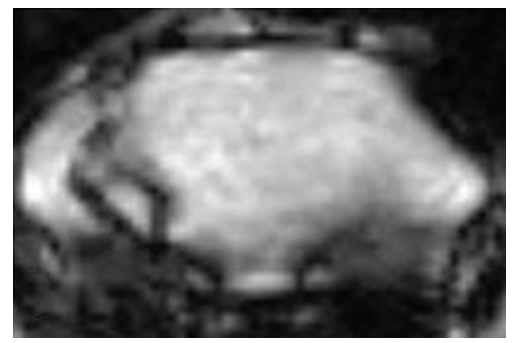

(i)

Figure 3: Temporal evolution of hematoma and edema after day 1 (a, b, and c), day 3 (d, e, and f), and day 10 (g, h, and i). On T1-weighted images, the hyperintense hematoma on day 1 (a) gradually changes to a hyperintense core over 10 days (c). On T2-weighted images, the core of the hematoma becomes hyperintense within 3 days (e) and develops a hypointense ring on day 10 (h) representing cavitation. The latter could also be discerned on T2* -weighted images on day 10 (i).

possible to suppress this artifact completely. For T1-weighted sequence, the echo time was minimized to remove any $\mathrm{T} 2$ weighting and minimize susceptibility artifacts at air/tissue interfaces. In T2-weighted sequence, a RARE factor of 12 increased the effective echo time and reduced the scan time. The echo time in $\mathrm{T}^{*}$ sequence was optimized to provide adequate signal-to-noise ratio while maximizing the contrast for measurement of hematoma.

A review of the literature revealed only two reports $[13,27]$ with murine experimental ICH and quantitation of hematoma size on $\mathrm{T}^{*}{ }^{[13]}$ alone or $\mathrm{T} 2$-weighted images [27]. Given these extremely limited data on the changes in signal intensities in the course of hematoma resorption and the lack of data for edema formation, we undertook this study to describe the signal characteristics of hematoma resorption and edema evolution in mice over time.

In our pilot study, we show for the first time that intracerebral hemorrhages appear hypointense on $\mathrm{T} 1$ at high field strength as early as 2 hours after the ictus. Although less well demarcated compared to $\mathrm{T} 2 *$ images, the hematoma remained discernable on $\mathrm{T} 1$ in the course of hematoma resorption. Hematoma volumes on T1 correlated well with those on histology $(P<0.04)$. T2 images approximated edema size well in the acute to chronic phase of edema formation in mice when compared with histology $(P<0.03)$. Van der Weerd et al. [28] had found T2-weighted images useful for quantitation of lesion volume 24 hours after stroke in a MCAO model. As our results indicate, T2* changes do not correlate with hematoma volumes on histology. That might be due to blooming artifacts that render the suitability of T2* images for quantification of hematoma at this field strength questionable [13]. Knight et al. [29] assessed the evolution of intracerebral hemorrhage in rats by MRI estimates of $\mathrm{T} 2$ relaxation time and hematoma-induced changes in cerebral blood flow and blood-brain barrier permeability over 14 days using a 7-Tesla, $20-\mathrm{cm}$ bore magnet. The lesion core and adjacent rim were identified by windowing of $\mathrm{T} 2$ values and changed in a consistent manner over time. The MRI and histological estimates of tissue loss were well correlated.

Despite the novel approach for following the temporal and spatial resolutions of intracerebral hemorrhages in mice by high field MR imaging, our pilot study has several limitations. The small number of animals at different examination dates precludes subgroup analysis. The high costs for experimental MR imaging at high field are a major drawback to 


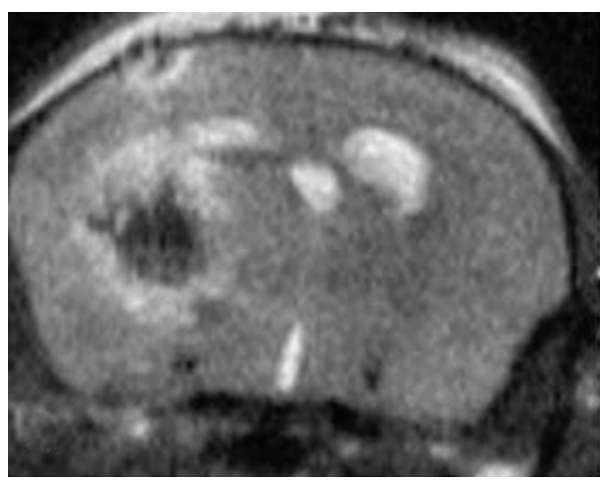

(a)

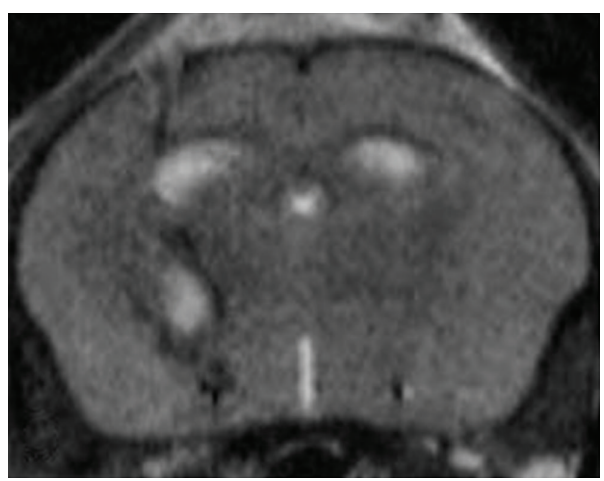

(c)

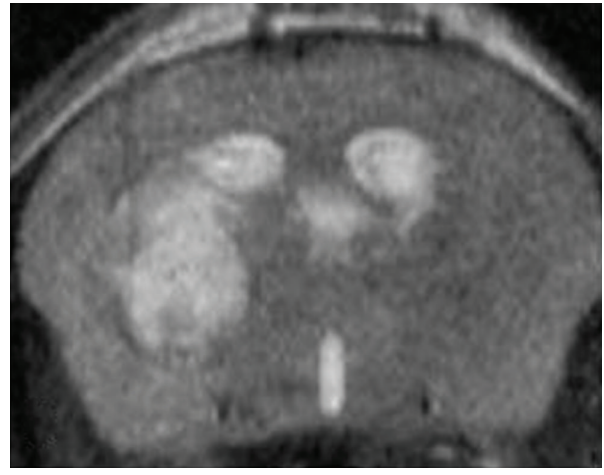

(b)

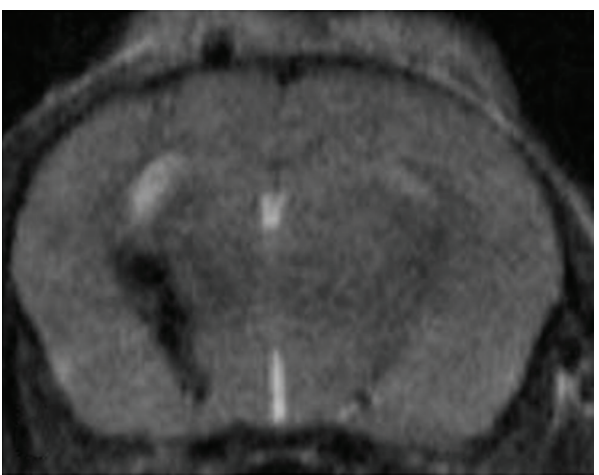

(d)

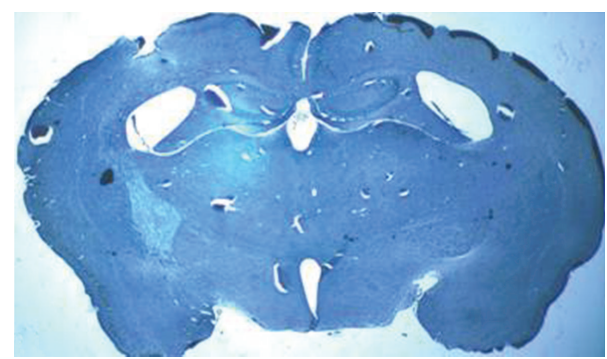

(e)

FIGURE 4: T2-weighted RARE spin-echo images at 24 hours (a) show predominantly low-signal hematoma within the left caudate/putamen, consistent with deoxyhemoglobin and intracellular methemoglobin. At 72 hours (b), there is conversion to predominantly high-signal, consistent with extracellular methemoglobin. In the course of hematoma resorption, the core hyperintensity (c) subsequently transforms to a hypointense gliotic scar at 21 days $(\mathrm{d})$. The cryostat section stained with Luxol Fast Blue and cresyl violet [×10] (e) also well delineates the gliotic scar.

large study groups. The reproducibility of the murine $\mathrm{ICH}$ model used in our study that was pioneered by Clark et al. [14] made it one of the principal models of murine $\mathrm{ICH}$ $[11,12,15-17,30]$. It mimics the pathogenetic mechanism of vessel wall rupture leading to intracerebral hemorrhage. Theoretical concerns that bacterial collagenase might induce an inflammatory response, independent of that elicited by parenchymal blood, have not been confirmed for the activation of microglia in cell cultures after the addition of collagenase at various concentrations [16]. Whether the morphological substrate of what we depict as hyperintensity on T2-weighted images exclusively represents edema, remains questionable. Alternate techniques imply the injection of autologous blood into the striatum $[8,10]$, but lack the pathophysiologic event of vessel rupture.

\section{Conclusions}

Repetitive multimodal MRI scanning at $4.7 \mathrm{~T}$ in mice with collagenase-induced ICH allows studying the individual kinetics of signal intensities underlying hematoma resolution and edema formation in a noninvasive manner. That would allow studying a wide range of therapeutic treatments in a longitudinal fashion in mice. 


\section{Acknowledgment}

This paper was supported by National Nature Science Foundation of China (81171112, MC Li).

\section{References}

[1] A. I. Qureshi, S. Tuhrim, J. P. Broderick, H. H. Batjer, H. Hondo, and D. F. Hanley, "Spontaneous intracerebral hemorrhage," The New England Journal of Medicine, vol. 344, no. 19, pp. 1450-1460, 2001.

[2] G. Xi, R. F. Keep, and J. T. Hoff, "Mechanisms of brain injury after intracerebral haemorrhage," The Lancet Neurology, vol. 5, no. 1, pp. 53-63, 2006.

[3] C. M. Miller, P. M. Vespa, D. L. McArthur, D. Hirt, and M. Etchepare, "Frameless stereotactic aspiration and thrombolysis of deep intracerebral hemorrhage is associated with reduced levels of extracellular cerebral glutamate and unchanged lactate pyruvate ratios," Neurocritical Care, vol. 6, no. 1, pp. 22-29, 2007.

[4] J. Castillo, A. Davalos, J. Alvarez-Sabin et al., "Molecular signatures of brain injury after intracerebral hemorrhage," $\mathrm{Neu}$ rology, vol. 58, no. 4, pp. 624-629, 2002.

[5] T. D. Ardizzone, A. Lu, K. R. Wagner, Y. Tang, R. Ran, and F. R. Sharp, "Glutamate receptor blockade attenuates glucose hypermetabolism in perihematomal brain after experimental intracerebral hemorrhage in rat," Stroke, vol. 35, no. 11, pp. 2587-2591, 2004.

[6] K. R. Wagner, "Modeling intracerebal hemorrhage: glutamate, nuclear factor-kappa B signaling and cytokines," Stroke, vol. 38, no. 2, pp. 753-758, 2007.

[7] S. T. Carmichael, P. M. Vespa, J. L. Saver et al., "Genomic profiles of damage and protection in human intracerebral hemorrhage," Journal of Cerebral Blood Flow and Metabolism, vol. 28, no. 11, pp. 1860-1875, 2008.

[8] J. Wang, J. Fields, and S. Dore, "The development of an improved preclinical mouse model of intracerebral hemorrhage using double infusion of autologous whole blood," Brain Research, vol. 1222, pp. 214-221, 2008.

[9] R. F. Regan, M. Chen, Z. Li, X. Zhang, L. Benvenisti-Zarom, and J. Chen-Roetling, "Neurons lacking iron regulatory protein-2 are highly resistant to the toxicity of hemoglobin," Neurobiology of Disease, vol. 31, no. 2, pp. 242-249, 2008.

[10] M. Xue, M. D. Hollenberg, and V. W. Yong, "Combination of thrombin and matrix metalloproteinase- 9 exacerbates neurotoxicity in cell culture and intracerebral hemorrhage in mice," Journal of Neuroscience, vol. 26, no. 40, pp. 1028110291, 2006.

[11] D. W. Kim, S. H. Im, J. Y. Kim, D. E. Kim, G. T. Oh, and S. W. Jeong, "Decreased brain edema after collagenase-induced intracerebral hemorrhage in mice lacking the inducible nitric oxide synthase gene: laboratory investigation," Journal of Neurosurgery, vol. 111, no. 5, pp. 995-1000, 2009.

[12] E. Titova, C. G. Kevil, R. P. Ostrowski et al., "Deficiency of CD18 gene reduces brain edema in experimental intracerebral hemorrhage in mice," Acta Neurochirurgica, Supplementum, no. 105 , pp. 85-87, 2008.

[13] S. Illanes, W. Zhou, S. Heiland, Z. Markus, and R. Veltkamp, "Kinetics of hematoma expansion in murine warfarinassociated intracerebral hemorrhage," Brain Research, vol. 1320, pp. 135-142, 2010.

[14] W. Clark, L. Gunion-Rinker, N. Lessov, and K. Hazel, "Citicoline treatment for experimental intracerebral hemorrhage in mice," Stroke, vol. 29, no. 10, pp. 2136-2140, 1998.
[15] R. Thiex, L. Mayfrank, V. Rohde, J. M. Gilsbach, and S. A. E. Tsirka, "The role of endogenous versus exogenous tPA on edema formation in murine ICH," Experimental Neurology, vol. 189, no. 1, pp. 25-32, 2004.

[16] J. Wang, A. D. Rogove, A. E. Tsirka, and S. E. Tsirka, "Protective role of tuftsin fragment 1-3 in an animal model of intracerebral hemorrhage," Annals of Neurology, vol. 54, no. 5, pp. 655-664, 2003.

[17] J. Wang and S. E. Tsirka, "Tuftsin fragment $1-3$ is beneficial when delivered after the induction of intracerebral hemorrhage," Stroke, vol. 36, no. 3, pp. 613-618, 2005.

[18] S. Geisler, H. Heilmann, and R. W. Veh, "An optimized method for simultaneous demonstration of neurons and myelinated fiber tracts for delineation of individual trunco- and palliothalamic nuclei in the mammalian brain," Histochemistry and Cell Biology, vol. 117, no. 1, pp. 69-79, 2002.

[19] J. J. Ohab, S. Fleming, A. Blesch, and S. T. Carmichael, "A neurovascular niche for neurogenesis after stroke," Journal of Neuroscience, vol. 26, no. 50, pp. 13007-13016, 2006.

[20] C. Foerch, K. Arai, G. Jin et al., "Experimental model of warfarin-associated intracerebral hemorrhage," Stroke, vol. 39, no. 12, pp. 3397-3404, 2008.

[21] B. Orakcioglu, K. Becker, O. W. Sakowitz, A. Unterberg, and P. D. Schellinger, "Serial diffusion and perfusion MRI analysis of the perihemorrhagic zone in a rat ICH model," Acta Neurochirurgica, Supplementum, no. 103, pp. 15-18, 2008.

[22] R. Thiex, W. Kuker, H. D. Muller et al., "The long-term effect of recombinant tissue-plasminogen-activator (rt-PA) on edema formation in a large-animal model of intracerebral hemorrhage," Neurological Research, vol. 25, no. 3, pp. 254-262, 2003.

[23] R. Thiex, J. Weis, T. Krings et al., "Addition of intravenous Nmethyl-D-aspartate receptor antagonists to local fibrinolytic therapy for the optimal treatment of experimental intracerebral hemorrhages," Journal of Neurosurgery, vol. 106, no. 2, pp. 314-320, 2007.

[24] H. Benveniste, K. Kim, L. Zhang, and G. A. Johnson, "Magnetic resonance microscopy of the C57BL mouse brain," NeuroImage, vol. 11, no. 6 I, pp. 601-611, 2000.

[25] R. F. Kooy, M. Verhoye, V. Lemmon, and A. van der Linden, "Brain studies of mouse models for neurogenetic disorders using in vivo magnetic resonance imaging (MRI)," European Journal of Human Genetics, vol. 9, no. 3, pp. 153-159, 2001.

[26] S. Xu, E. K. Jordan, W. Li et al., "In vivo three-dimensional MR microscopy of mice with chronic relapsing experimental autoimmune encephalomyelitis after treatment with insulinlike growth factor-I," The American Journal of Neuroradiology, vol. 19, no. 4, pp. 653-658, 1998.

[27] M. L. James, P. M. Sullivan, C. D. Lascola, M. P. Vitek, and D. T. Laskowitz, "Pharmacogenomic effects of apolipoprotein e on intracerebral hemorrhage," Stroke, vol. 40, no. 2, pp. 632-639, 2009.

[28] L. van der Weerd, M. F. Lythgoe, R. A. Badin et al., "Neuroprotective effects of HSP70 overexpression after cerebral ischaemia-an MRI study," Experimental Neurology, vol. 195, no. 1, pp. 257-266, 2005.

[29] R. A. Knight, Y. Han, T. N. Nagaraja et al., "Temporal MRI assessment of intracerebral hemorrhage in rats," Stroke, vol. 39, no. 9, pp. 2596-2602, 2008.

[30] T. Nakamura, G. Xi, Y. Hua, T. Schallert, J. T. Hoff, and R. F. Keep, "Intracerebral hemorrhage in mice: model characterization and application for genetically modified mice," Journal of Cerebral Blood Flow and Metabolism, vol. 24, no. 5, pp. 487494, 2004. 


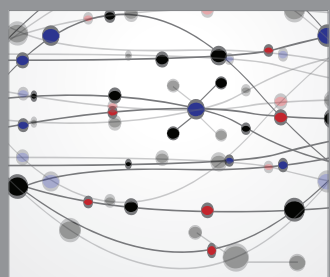

The Scientific World Journal
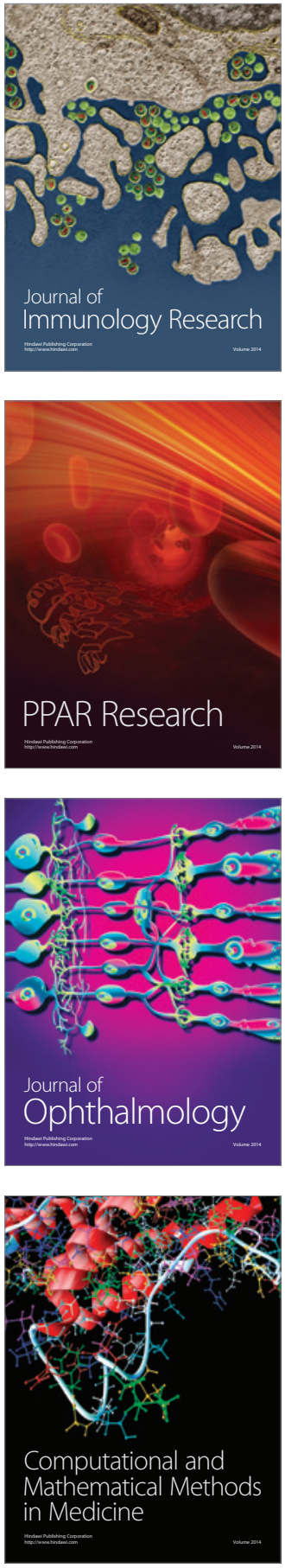

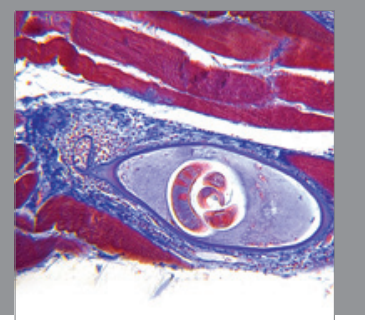

Gastroenterology

Research and Practice
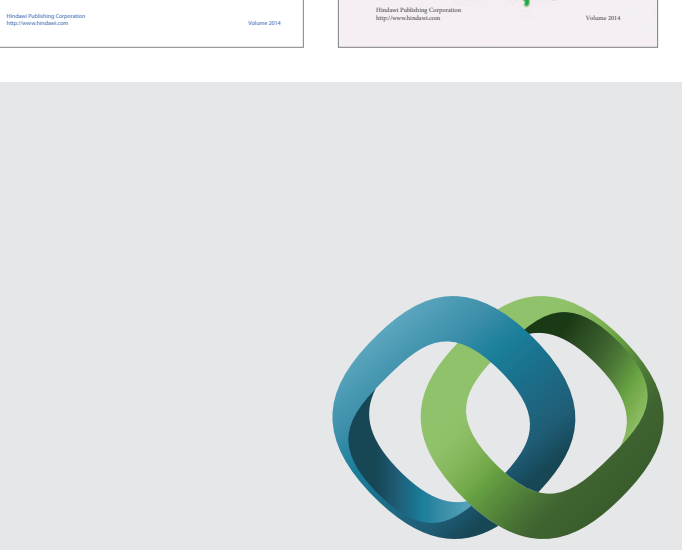

\section{Hindawi}

Submit your manuscripts at

http://www.hindawi.com
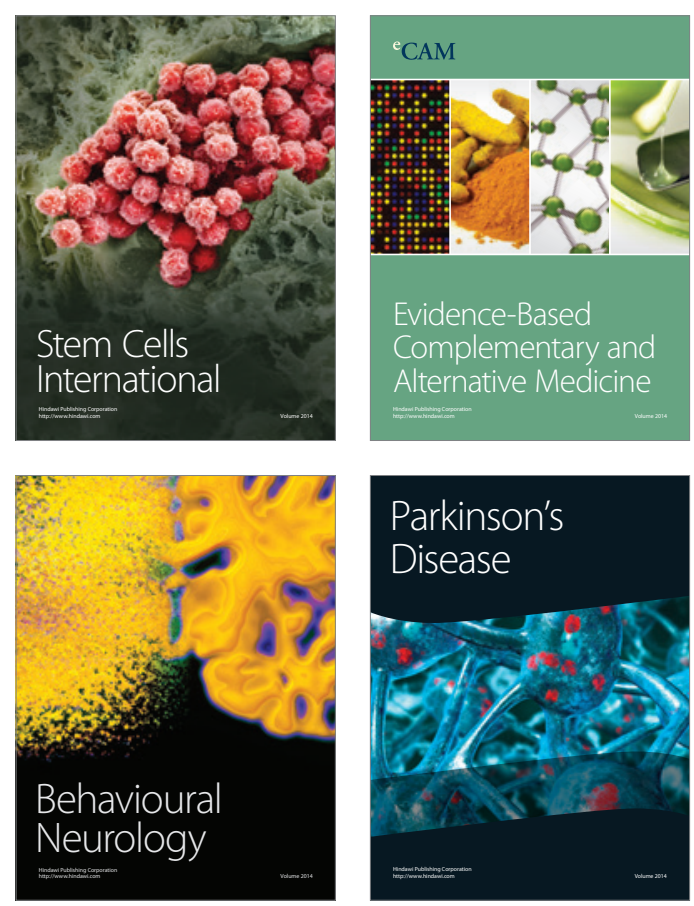

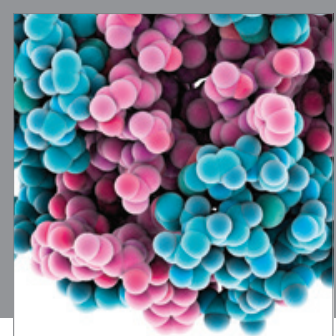

Journal of
Diabetes Research

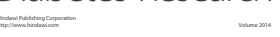

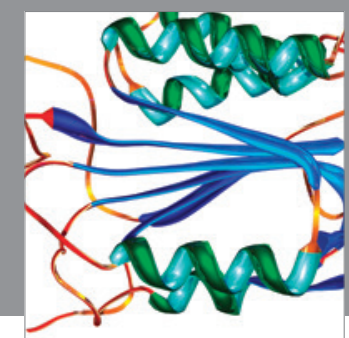

Disease Markers
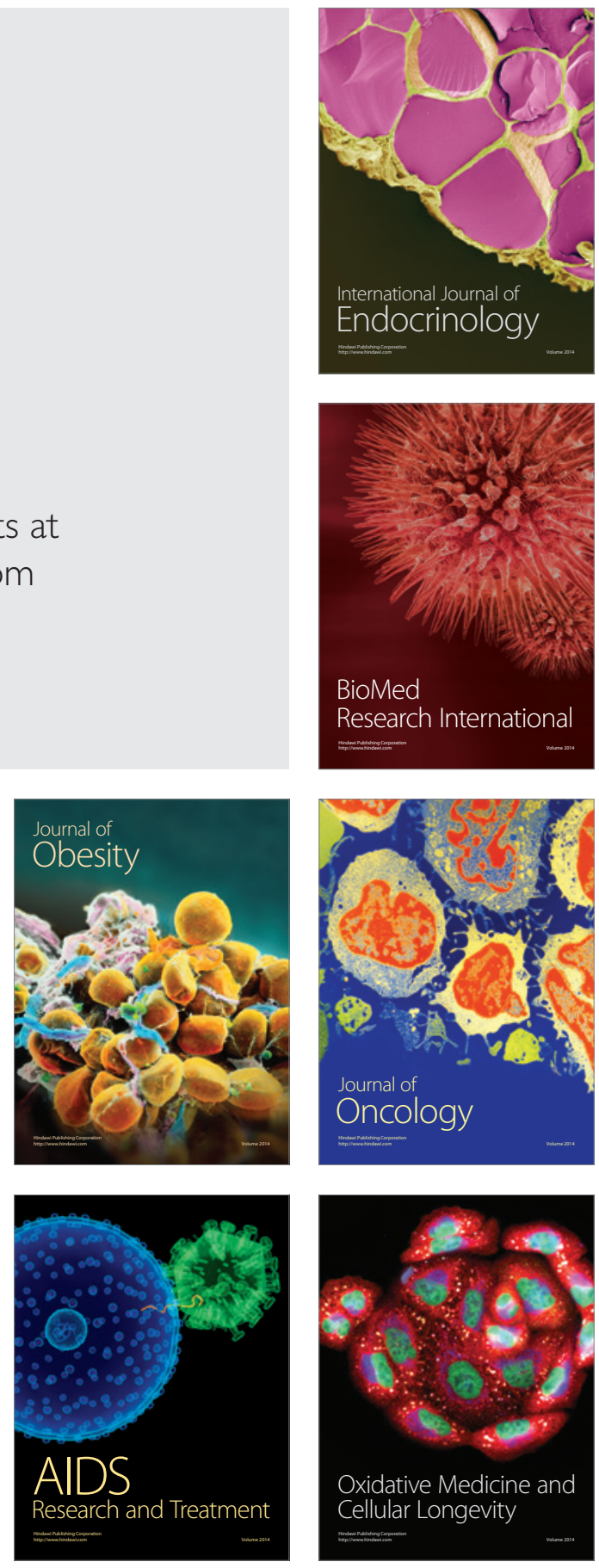